externally, and at one other spot through the walls of the vagina. This case, which my friend Mr. Nunn saw with me, as well as Dr. Locock, proved, on tapping, to be filled with a thick, cheesy matter, mixed with pus and hair, which escaped with great difficulty through the canula. But we have also distinct solid tumours in connexion with these fluid ones, and then there is no sense of fluctuation. This observation applies both before and after evacuating the contents of the fluid cysts.

The method of diagnosis, by means of a microscopic examination of the fluid withdrawn by paracentesis, deserves careful consideration. Dr. Jolnn Hughes Bennett, in his paper on ovarian disease, published in the Edinburgh Medical and Surgical Journal, expresses an opinion that it is of great value, and seems disposed to rely, to a very considerable extent, upon the indications so derived. In conjunction with my friend, Mr. T. W. Nunn, I have, during the past three years, given considerable attention to this point. The following are the conclusions at which he has arrived, and to which, I may add, I give my entire concurrence. Mr. Nunn this expresses himself:- "The fact that fluid withdrawn from the cavity of the abdomen by the operation of paracentesis may be, in one instance, the result of transudation of the serous part of the blood, in consequence of obstructed portal circulation; in another, the product of inflammatory action of the peritonæum; in another, a part of the contents of an hydatid; and in another, the distending secretion of an ovarian crst, might lead one to conceive the characteristics of each of these different fluids would be such as should enable one to docide at once upon the source from which each was derived, and that therefore the nature of the fluid would be diagnostic of the disease which gave rise to its production.

"In the present state of our knowledge, I do not think we are justified in asserting that such is the case. What $I$ believe to be the value of a microscopic examination of the fluid is, that it may serve to strengthen an opinion, but alone it ought not to decide one. As an illustration of what I mean, I would instance a somewhat analogous example: the presence of the prismatic crystals of the triple phosphate in the urine indicates the existence of a morbid condition that may be either a local disease or a general disorder; a knowledge of the other symptoms is required before it can be determined which of the two maladies is present; to be in possession of the fact of there being that peculiar deposit in the urine is, notwithstanding, of great importanoe.

"We must take into consideration these two points:-

"First. What does the microscope reveal that is peculiar in fluid of an ovarian cyst.

"Second. What are the fallacies to which a diagnosis founded upon a microscopic examination of the fluid is obnoxious.

"In respect of the first of these questions, I am inclined to say, as the result of many examinations of different specimens of ovarian fluid, that the most constant characteristic of such fluid is its containing in greater or less abundance cells gorged with granules, and, in addition, circumambient granules, hav. ing the same measurements as those encompassed by the cell wall. At one time, I considered the size of these granules (if they can properly be so called) was constant, but subsequent observations have convinced me of the incorrectness of this conclusion-the size of the gorged cells and of the granules varies greatly even in the fluids from different cysts of the same ovary.

"It would be foreign to the subject to enter into a description of all the occasional contents of ovarian cysts, and I therefore only refer the reader to the annexed engraving, which will in some measure illustrate the idea I wish to convey respecting the gorged cells and granules.
FIG. 1.

FIG. 2.

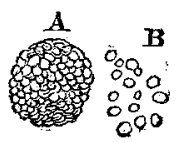

"A, in both figures, represents the gorged cells.

" $\mathrm{B}$, similarly, the granules.

"In Fig. 2, it will be seen that both the cells and the granules are formed on a much smaller scale than in Fig. 1.

"These drawings were made from specimens obtained from a multilorular encysted ovary, taken from a subject sent to the dissecting rooms of Middlesex Hospital. The fuids of the different cysts were carefully kept distinct.
"With regard to the second question, I would urge, in the first place, that the phenomena of cell growth are at best but imperfectly investigated, especially as bearing upon the phy. siology of cells which owe their existence to a morbid action, and that besides this, under certain circumstances, the ovarian fluid may not be contained within a cyst, as, for instance, where the cyst has been at some time or other ruptured, but may be mingled with peritonitic effusion, or the ordinary fluid of ascites; and, moreover, we must recollect that lymph and pus are not uncommonly found within an ovarian crst."

Having ascertained the nature of the tumour so far as to say whether it is unilocular or multilocular, the next important subject is, as to the presence of adhesions.

In examining for adhesions, I direct the patient should be laid in the horizontal posture, and made to flex the thighs on the abdomen, so as to relax the abdominal parietes. I then move the cyst from side to side. If this be readily done, know there are no adhesions. Again, I place my hand firmly on the relaxed parietes, and move them over the cyst; if they move readily, I know there are no adhesions on the upper and lateral surfaces of the cyst. Again, as the parietes are thin in this disease, I grasp and pucker them up, and then move them over the cyst, and also see if they gather up readily without raising the cyst itself. If $I$ find these three indications, I determine there are no adhesions. Anothez plan, for which I am indebted to my friend, Dr. Sibson, is based on the extent to which the contents of the abdomen are forced downwards during a deep inspiration, by the descent of the diaphragm. If there be no adhesions in front, the upper boundary of the ovarian tumour descends to the ex. tent of an inch during a deep inspiration, the place previously occupied by the tumour being now taken up by the intestines; consequently, if you percuss over the upper part of the tumour a dull sound is elicited during ordinary respiration, but when the patient takes a deep inspiration, an intestinal resonance is there perceptible.

(To be continued.)

Oxford-square, Hyde-park, March, 1850.

\section{ON THE USE OF NUX VOMICA AS A REMEDY IN HAY-FEVER.}

By G. T. GREAM, EsQ., M.R.C.S., \&c.

THE efficacy of nux vomica as a remedy for hay-fever is not generally known.

The symptoms which are removed by its administration are, distressing paroxysms of sneezing prolonged until a state of depression ensues, and preceded by excessive irritation of the Schneiderian membrane, the conjunctivx, and the face; the latter being heated at the time of the paroxysm, and during the intervals, as if a hot plate of iron was nearly in contact with it.

I believe that the farina of grass has no more influence ia causing the disease than that of any other flowers; in hay fields and hay-lofts there is necessarily more dust than elsewhere-but the dust from beaten carpets, from the roads, and from other sources, produces the same distressing symptoms. I am led to think that in the middle of summer, from the end of May to the end of July, at which time hay-fever generally ceases, a quantity of fine dust floats in the atmo. sphere, finer than any which is in the air at other seasons, increased probably by the farina of the mass of flowers at that period in bloom, but that during later and earlier months, the more frequent rains, and the dews at night, prevent these particles from leaving the ground; and I have been induced to suppose that this reasoning is correct, by finding that, however distressing the symptoms have been during the day, they are all entirely removed upon the occurrence of a shower of rain; the face becomes cool; the irritation of the nostrils and of the eyes ceases, and does not return until the heated atmosphere has again evaporated the fallen rain.

The relief afforded by a residence near the sea appears to be owing to the absence of fine particles of matter near so large a surface of water.

I am indebted to my friend Mr. Hammerton, of St. George's Hospital, for suggesting to me the nux romica as a remedy in this complaint, which has frequently caused me, personally, much annoyance; it was administered by a friend of his to large numbers of the country people in his neighbourhood, who flocked to him annually for relief, having experienced so much benefit from it

Having taken it for three years with decided effect, and 
having for nearly that time prescribed it for others, with equal success, I feel bound to publish it through your columns, if your will do me the favour to insert this letter, the results of its use in a harassing disorder, with which many percons are at this time threatened.

The preparation recommended, and which $I$ have always prescribed, is the tincture of nux vomica of the "Dublin Pharmacopoia." Ten drops of this should be given for a dose, in water, and increased gradually to twenty drops, three times a day: the action of it should at first be watched. It is an agreeable light bitter; increases the appetite; and influences the Schneiderian membrane, no doubt through the medium of the nerves.

I have accompanied the administration of the tincture with the application of an ointment (as high up in the nostrils as possible) composed of one drachm and a half of Goulard's ex tract; two ounces of spermaceti cerate, and a few drops of oil of roses or of bergamot.

Hertford-street, May-Fair, May 24, 1850.

\section{REPORT OF A CASE OF}

\section{STRANGULATED INGUINAL HERNIA, COM- PLICATED BY IMPERFECT DESCENT OF THE TESTICLE.}

\section{By H. FEATHERSTONHAUGH, Esฉ., \\ SURGEON TO THE GATESHEAD DISPENSARY}

ON the 14 th of January, 1850, William S-, aged twenty one, an athletic young man, a miller, was suddenly attacked with severe pain and tenderness in the epigastric and umbilical regions, accompanied by a sense of tightness and distention of the abdomen, with inability to evacuate. Some years previously, Mr. Annandale, of Newcastle, was consulted, i being supposed by his parents that he had a rupture on the right side. Mr. A., however, detected that it was the right testicle in the canal, and explained to them the needlessness of his wearing a truss. Since then he has had repeated attacks of constipation and obstruction of the bowels, which al ways yielded in two or three days to purgatives, injections, \&c.

On examination, I discovered that the right testicle had never descended into the scrotum, but could be felt just below the external ring, the tumour above having a soft feel. No impulse was felt on coughing. He was also snffering from gonorrhoa, and a suppurating sympathetic bubo on the same side.

Jan. 15th.-.Symptoms unabated.

I6th.- Still suffered from severe pain and distention of abdomen; constant vomiting of dark fluid; hiccup; and pain over tumour, with complete obstruction of the bowels, notwithstanding the treatment he had undergone,-Twelve o'clock, A.M.: Had a consultation with Mr. George Heath, and deter: mined upon performing the operation, in which he assisted me. An incision was made along the course of the inguinal canal, and the dissection carried down to the sac at its lower part; in doing so the upper part of the bubo was laid open, and a quantity of pus let out. On opening the sac just below the external ring, a good deal of fluid escaped and omentum, of a dark and thickened appearance, covering the body of the testicle, which was small and healthy, firmly adherent, and bronght into view. On enlarging the incision, and opening the canal upwards towords the internal ring, the epididymis tras observed, and in carrying the finger up to the internal ring a very small portion of gut could be felt protruding and strangulated at this point. The stricture was then divided, and the intestine returned into the abdomen. The greater part of the omentum was cut off, and a ligature applied to one or two bleeding vessels, the rest being allowed to remain in contact with the testicle, which had retracted slightly in the canal; wound dressed by means of sutures.-Six o'clock P.M.: Surface of body warm; abdomen softer; freer from pain; had slept a little; pulse fuller; hiccup and vomiting had ceased. Ordered a draught with tincture of hoscyamus, and an enema with castor oil, and warm water.

17 th.-Less pain and distention of abdomen; bowels had acted; complained of severe pain in the loins; pulse 105, and full; slight hiccup; no vomiting; thirst; had slept occasionally during the night. Continue enemas, with calomel, two grains, powdered opium, quarter of a grain, every four hours.

18th.-Distention and pain of abdomen less; hiccup ceased; palse 100; thirst less urgent; tongue cleaner; bowels had been moved freely; he had passed a quantity of urine; wound looked well. Continue powders and enemas.

19th.- No pain except in the loins; distention of abdomen less; pulse 90; thirst abating; bowels open; a good deal of swelling and inflammation of the wound. Continue injections, with linseed ponltices to the part.

20th.-Complained of severe pain in the loins; pulse 86; tongue cleaner; slight thirst; he wished to have food; bowels had been moved; free from pain and tension of the abdomen; uneasiness in the wound about the testicle.

21st.- Still better; pulse 80; no thirst; bowels open; pain and distention ceased; appetite improved; wound suppurating freely. Continue enemas, poultices, and castor oil, occasionally, with beef-tea.

29 th.-Going on well, and wound healing.

April 1st.- Has progressed satisfactorily from this time, and is now quite well.

Newcastle-on-Tyne, April, 1850.

\section{ON THE ELECTRIC AND MAGNE'TIC FLUIDS.}

\section{Bx W. F. STEVENSON, EsQ., F.R.S.}

THE phenomena apparent in the electric and magnetic fluids -..viz., the positive and negative qualities of the former, and the attractive and repulsive qualities of the latter, as well as the direction of the magnetic needle to the north pole-are not the effects of any peculiar properties in those fluids, but are due solely to the circumstance that the two fluids are in perpetual motion (when free) upon the earth's surface, and that they progress invariably in the direction of the Arctic pole.

These theorems are proved by the following experiments:1st. Let $a-b$ be a brass-cylindrical rod, suspended by a non-conducting or silken string, or placed upon an isolated table, and then receive the electric fluid at the end $b$, when such fluid will be immediately found to have passed from $b$ to the other extremity of the rod $a$, from whence it will continually escape, and be ultimately carried off by the slower conducting power of the atmosphere.

If any number of similar isolated rods be in like manner made to receive the fluid at the end $b$, and are then connected together with the first rod by approaching the end $a$ of one rod to the end $b$ of the preceding one, the fluid of all the rods will be found to have passed on to the extremity $a$ of the first rod. That the fluid which has so passed on to the end $a$ of the first rod, and is there making its exit, will be found to be positive (as it is now called), whilst the other extremity of the rods will be found to be negative-the positive end being that where the exit of the fluid takes place, and the negative end that where it entered, and thus forming together a current of electricity.*

That the phenomena of the positive and negative qualities of the fluid are the result of the movement of the fluid along the rods, will be evident on reversing the first rod, and placing the end $\alpha$ (where the fluid is escaping) in connexion with the end $a$ of the second rod, when the current will be necessarily interrupted, and from which circumstance there will be found no positive electricity at the latter extremity of the rods. It will be seen, from what I have stated, that, as the electric fluid is in perpetual motion, and that in its free state it is constantly passing over the earth's surface in one direction, a good conductor must be such an instrument as will best facilitate the transit of the fluid, or enable it to join the general current whenever it is met with detached from such current. Now, although a metal is the best material for a conductor, its power of conduction depends nevertheless upon its shape. For instance, if the fluid is to be conducted or transferred from any body where it may be found into the general current, it is evident that a metal in a circular form, or that of a ball, caunot facilitate such transfer; that, on the contrary, the fluid upon metal of such a shape must be, in a manner, confined and imprisoned, and thus prevented from passing into the general current.

It is this circumstance which leads to the use of small globes at the ends of metallic conductors, when it is desirable to retain the fluid, and that conductors with pointed ends are those which best allow of the exit of the fluid.

It follows, from what $I$ have advanced, that the negative fluid, as it is called, is only the ordinary fluid when met with upon a non-conducting substance, or upon a surface which,

* "Electricity is the only element which is constantly in motion unless restrained; the only one, therefore, th imay be said to possess 